\title{
Calliandra calothyrsus: A Potential Host for the Indian Lac Insect [Kerria lacca (Kerr.)] Cultivation in India
}

\author{
A. Mohanasundaram, K.K. Sharma, V.D. Lohot, T. Kandasamy, Upnit Shree and \\ Naaserah Zeeshan \\ Lac Production Division, ICAR-Indian Institute of Natural Resins and Gums, Namkum, Ranchi-834 010, India \\ Email: mohaniinrg@gmail.com
}

\begin{abstract}
More than 400 host plants have been reported to support lac insects throughout the world but not all have been exploited for their commercial potential except some traditional hosts. In this study, potentially of four host plants viz., Calliandra calothyrsus, Calliandra surinamensis, Dalbergia assamica and Malvaviscus penduliflorus was evaluated for kusmi and rangeeni strain of Kerria lacca (Kerr.) during three consecutive years 2014-15, 2015-16 and 2016-17. The data on pre and post-harvest parameters viz., initial settlement density, mortality, fecundity, cell weight, resin weight, broodlac, broodlac ratio and total scrapedlac were recorded based on pre and post-harvest attributes of rangeeni (summer season (baisakhi) \& rainy season (katki) crop) and kusmi summer season (jethwi) \& winter season (aghani). C. calothyrsus outperformed other hosts during all the three years. In addition to this, $C$. calothyrsus is having quick growth, bushy nature and high response to coppicing make this host ideal for Lac Integrated Cropping System (LICS).
\end{abstract}

Keywords: Calliandra calothyrsus, Kerria lacca, Rangeeni, Kusmi

Indian lac insect, Kerria lacca (Kerr.) belongs to the family Tachardiidae (=Kerridae), order Hemiptera and superfamily Coccoidea is phytosuccivorous and sessile. The presence of lac insects was first reported on Ficus religiosa L. and Ficus indica L. (now F. benghalensis) along with Butea monosperma Lam (palas), Ziziphus mauritiana Lamk (ber) from Bihar region (Now Bihar, Jharkhand and some parts of West Bengal). More than 400 plant species are reported as a host for lac insects from different parts of the country. There are two strains of the Indian lac insect viz., rangeeni and kusmi. Each strain completes its life-cycle twice a year with varying period. Of the two strains i.e., kusmi and rangeeni, aghani (winter season) crop of kusmi strain contributes the most to the total lac production (Sharma and Ramani 1999). Lac insect strains have been generally characterized by different life-cycle and their respective host plants. Lac production and quality are dependent on the host plant and the strain of lac insect. Besides lac host plant, $K$. lacca is associated with large pest complex comprising predatory and parasitic insects which influence lac production and quality. Various parasitoids such as Aprostocetus purperous (Cam.), Trachidaephagus trachidae (How.) are responsible for majority of crop loss. Among these parasitoids, $A$. purperous alone has acquired the most dreaded pest of lac insect (Mohanasundaram et al 2016). Lac tree=Kusum (Schleichera oleosa Lour.), Indian jujube=ber (Ziziphus mauritiana Lamk.) and Flame of forest=palas (Butea monosperma Lam.) are major lac hosts, however these host plants have some constraints like longer gestation period to raise new plantation (5 to 10 years), longer waiting period after pruning like kusum (12-18 months), strain specificity i.e. only kusmi strain on kusum and rangeeni on palas and difficulty in lac culture operation due to a few morphological characters like thorns on ber. Studies have been conducted earlier on exploring the bushy host plants so that they can be integrated with the agricultural crops. One such bushy host plant, Flemingia semialata Roxb. (Semialata) has gained popularity among farmers recently. Calliandra calothyrsus Meisn, Calliandra surinamensis Benth, Dalbergia assamica Benth and Malvaviscus arboreus var. penduliflorus (DC.) Schery has been reported as a lac insect host plants (Ramani and Sharma 2010, Sharma et al 2012). Looking into the major host plants, there is a need to identify more host plants which are easy to maintain from lac cultivation aspects. The lac host plants under study are bushy in nature and quick growing in habit. Therefore, the present study was undertaken to evaluate the potentiality of these hosts for lac cultivation under Ranchi conditions.

\section{MATERIAL AND METHODS}

Evaluation was carried out in the summer and rainy seasons of Rangeeni strain of lac insect and summer and winter (seasons of kusmi strain of lac insect on four bushy hosts viz., C. calothyrsus, C. surinamensis, D. assamica and 
M. penduliflorus during three consecutive years (2014-15 to 2016-17). Field experiments were undertaken at Institute Research Farm, ICAR- Indian Institute of Natural Resins and Gums (IINRG), Namkum, Ranchi, Jharkhand $\left(23.19^{\circ} \mathrm{N}\right.$ latitude and $85.22^{\circ} \mathrm{E}$ longitude and elevation 2076 feet). Seedlings were grown in paired row system in triangular method of planting by keeping four meter distance between plants, two meter distance between a paired row and four meter distance between two paired rows during the year 2012 (Fig. 1). Ten plants of each host were taken per season ( 5 for rangeeni lac insect and 5 for kusmi lac insect); in total 40 plants were used for this experiment. Required agronomic practices viz., weeding, earthing up, pruning, FYM and fertilizer application, pesticide application, irrigation etc. were followed for maintenance. Plants were pruned in the month of February-March for inoculation of winter season crop and in June-July for inoculating summer season crop of kusmi strain. Similarly, plants were pruned in the month of February-March for inoculation of rainy season crop and in April-May for inoculating summer season crop of rangeeni strain. Recommended packages of practices for lac cultivation were followed. Observations on biological and economic attributes of lac insect were recorded as per the standard procedure (Mohanasundaram et al 2016). Preharvest productivity parameters viz., settlement density (crawlers per sq cm), initial mortality (\%), sex ratio (\% male); post-harvest productivity parameters viz., fecundity (Nos./Female), cell weight (mg), resin weight (mg) and yield parameters viz., broodlac (includes both stick and lac insect cell weight) $(\mathrm{g})$, rejected lac $(\mathrm{g})$, total scrapedlac (without stick weight i.e only lac resin, dye and wax etc.) (g), broodlac ratio (output/input) were taken. The performance of all the four host plants was compared for all season crops of $K$. lacca. The experiment was conducted in Randomized Block Design. Three consecutive years' data were pooled. Field level evaluation of the best performing host in the study i.e. $C$. calothyrsus was also conducted at farmer's field at two different villages' viz., Lodama and Benyazara of Ranchi district, Jharkhand during 2017-20 to further ensure its potential for commercial lac cultivation. Lac cultures on stunted host plants during different seasons are (Figs. 2 to 5 ).

\section{RESULTS AND DISCUSSION}

Evaluation of $K$. lacca (rangeeni) during summer (baisakhi) crop: Though initial settlement density of $K$. lacca (rangeeni) on M. penduliflorus was more than the other hosts but lac insect mortality was observed before sexual maturation. Initial mortality was minimum in C. calothyrysis (12.55\%) and maximum in C. surinamensis (27.06\%) (Table 1). Sex ratio ranged between 46.89 to 64.20 per cent, whereas, lac insect mortality was observed on $M$. penduliflorus before sexual maturation. Average fecundity was significantly more on C. calothyrsus (337.5) followed by $D$. assamica and C. surinamensis. Cell weight (15.41 and $12.46 \mathrm{mg}$ ) and resin weight (12.51 and $9.95 \mathrm{mg}$ ) was maximum in $C$. calothyrsus and minimum in D.assamica, respectively. Yield attributes, viz., broodlac, rejected lac, total scrapedlac and broodlac ratio were significantly higher in $C$. calothyrsus $(1712.53,476.87 \mathrm{~g}, 126.33$ and $4.8 \mathrm{~g}$ per plant, respectively) than $C$. surinamensis and $D$. assamica. Monobrullah et al (2016) reported that the pre-harvest parameters of rangeeni lac insect viz., initial mortality, sex ratio and broodlac yield were significantly different between hosts berand palas. Initial mortality and sex ratio ranged from 12.86 to 47.85 per cent and 22.97 to 40.86 per cent, respectively and resin weight also significantly differed between these hosts during rangeeni summer season crop. Sharma and Jaiswal (2011) observed that, density of settlement of lac insect is on an average about 150 larvae settled in one square $\mathrm{cm}$ area. Depending upon the host-

Table 1. Bio-economical parameters of summer season (baisakhi) crop of rangeeni lac insect during 2014-2016 (Pooled)

\begin{tabular}{|c|c|c|c|c|c|c|c|c|c|c|}
\hline \multirow[t]{2}{*}{$\begin{array}{l}\text { Bushy host } \\
\text { plants }\end{array}$} & \multicolumn{3}{|c|}{$\begin{array}{l}\text { Pre-harvest productivity } \\
\text { parameters }\end{array}$} & \multicolumn{3}{|c|}{$\begin{array}{c}\text { Post-harvest productivity } \\
\text { parameters }\end{array}$} & \multicolumn{4}{|c|}{ Yield parameters } \\
\hline & $\begin{array}{c}\text { Settlement } \\
\text { density } \\
\left(\text { Nos. } / \mathrm{cm}^{2}\right)\end{array}$ & $\begin{array}{c}\text { Initial } \\
\text { mortality } \\
(\%)\end{array}$ & $\begin{array}{l}\text { Sex ratio } \\
\text { (\% Male) }\end{array}$ & $\begin{array}{c}\text { Fecundity } \\
\text { (nos./ } \\
\text { Female) }\end{array}$ & $\begin{array}{l}\text { Cell weight } \\
\text { (mg) }\end{array}$ & $\begin{array}{c}\text { Resin } \\
\text { weight } \\
(\mathrm{mg})\end{array}$ & $\begin{array}{l}\text { Broodlac } \\
\text { (g) }\end{array}$ & $\begin{array}{l}\text { Rejected } \\
\text { lac } \\
\text { (g) }\end{array}$ & $\begin{array}{c}\text { Total } \\
\text { scraped lac } \\
\text { (g) }\end{array}$ & $\begin{array}{l}\text { Broodlac ratio } \\
\text { (Output/ input) }\end{array}$ \\
\hline C. calothyrsus & $\begin{array}{l}90.53 \\
(9.52)\end{array}$ & $\begin{array}{l}12.55 \\
(3.60)\end{array}$ & $\begin{array}{l}53.26 \\
(7.33)\end{array}$ & $\begin{array}{l}337.53 \\
(18.38)\end{array}$ & $\begin{array}{l}15.41 \\
(3.99)\end{array}$ & $\begin{array}{l}12.51 \\
(3.61)\end{array}$ & $\begin{array}{l}1712.53 \\
(41.16)\end{array}$ & $\begin{array}{l}476.87 \\
(20.61)\end{array}$ & $\begin{array}{l}126.33 \\
(11.16)\end{array}$ & $\begin{array}{c}4.80 \\
(2.30)\end{array}$ \\
\hline C. surinamensis & $\begin{array}{l}47.18 \\
(6.90)\end{array}$ & $\begin{array}{l}27.06 \\
(5.25)\end{array}$ & $\begin{array}{l}64.20 \\
(8.03)\end{array}$ & $\begin{array}{l}127.69 \\
(11.28)\end{array}$ & $\begin{array}{l}14.66 \\
(3.89)\end{array}$ & $\begin{array}{l}11.65 \\
(3.48)\end{array}$ & $\begin{array}{l}527.00 \\
(22.67)\end{array}$ & $\begin{array}{l}511.33 \\
(19.67)\end{array}$ & $\begin{array}{l}16.22 \\
(3.81)\end{array}$ & $\begin{array}{c}1.40 \\
(1.38)\end{array}$ \\
\hline D. assamica & $\begin{array}{l}70.16 \\
(8.40)\end{array}$ & $\begin{array}{l}11.00 \\
(3.37)\end{array}$ & $\begin{array}{l}46.89 \\
(6.87)\end{array}$ & $\begin{array}{l}195.98 \\
(13.98)\end{array}$ & $\begin{array}{l}12.46 \\
(3.59)\end{array}$ & $\begin{array}{c}9.95 \\
(3.23)\end{array}$ & $\begin{array}{l}414.00 \\
(20.35)\end{array}$ & $\begin{array}{l}20.67 \\
(4.39)\end{array}$ & $\begin{array}{l}35.92 \\
(5.98)\end{array}$ & $\begin{array}{c}1.80 \\
(1.52)\end{array}$ \\
\hline M. penduliflorus & $\begin{array}{l}91.60 \\
(9.58)\end{array}$ & $\begin{array}{l}18.87 \\
(4.35)\end{array}$ & $\begin{array}{l}0.00 \\
(0.71)\end{array}$ & $\begin{array}{l}0.00 \\
(0.71)\end{array}$ & $\begin{array}{l}0.00 \\
(0.71)\end{array}$ & $\begin{array}{c}0.00 \\
(0.71)\end{array}$ & $\begin{array}{l}0.00 \\
(0.71)\end{array}$ & $\begin{array}{l}0.00 \\
(0.71)\end{array}$ & $\begin{array}{l}0.00 \\
(0.71)\end{array}$ & $\begin{array}{c}0.00 \\
(0.71)\end{array}$ \\
\hline$C D(p=0.05)$ & 0.66 & 0.73 & 0.50 & 0.95 & 0.24 & 0.23 & 3.56 & 10.56 & 1.67 & 0.11 \\
\hline
\end{tabular}

${ }^{*}$ Figures in parentheses are square root $\sqrt{ }(X+0.5)$ transformation values; Means are significant at $p<0.05$ 


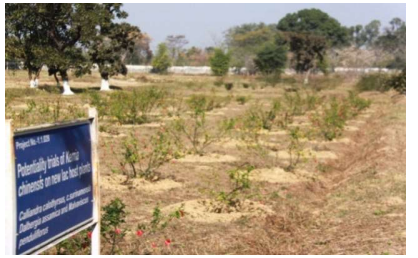

Malvaviscus penduliflorus

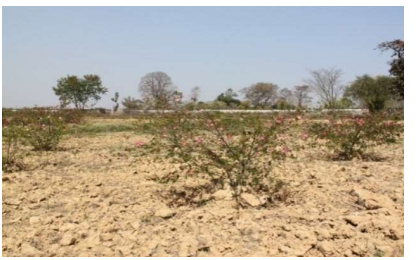

Calliandra surinamensis

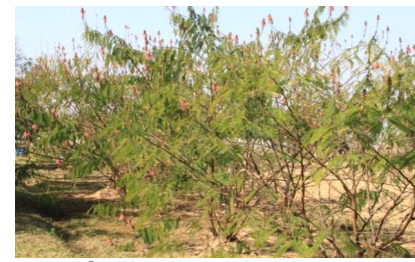

Calliandra calothyrsus

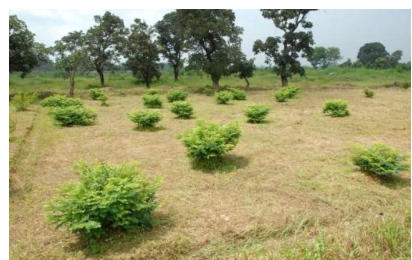

Dalbergia assamica

Fig. 1. Field view of bushy host plants at Institute Research Farm, IINRG, Ranchi

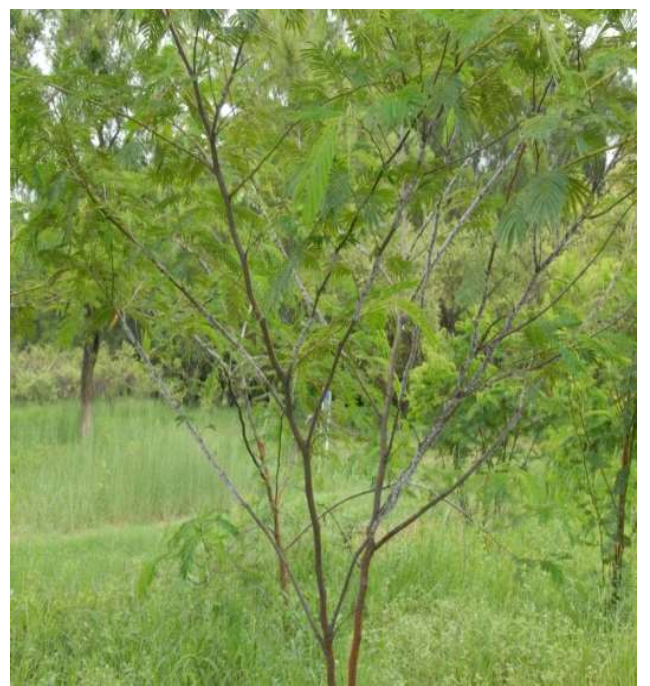

Fig. 2. K. lacca (rangeeni) on C. calothyrsus during summer (baisakhi) crop

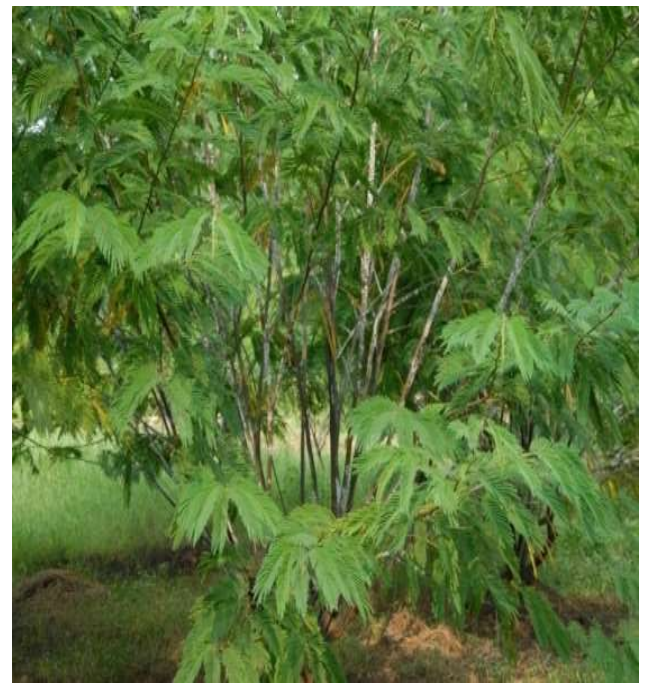

Fig. 3. K. lacca (rangeeni) on C. calothyrsus during rainy (katki) crop

plant and season of inoculation, carrying capacity of the host plant varies. Kumar et al (2007) concluded that fecundity of lac insects when grown on F. macrophylla was 338.0 and 460.8 nos. and resin weight ( 9.46 and $13.67 \mathrm{mg}$ ) during katki and baisakhi crops, respectively. The density of settlement of lac insect varied between 48.3 to 162.0 no. of crawlers $/ \mathrm{cm}^{2}$ and resin output ranged from 5.60 to $8.15 \mathrm{mg}$ per cell for summer crop of rangeeni strain on F. macrophylla (Gupta et al 2020).

\section{Evaluation of $K$. lacca (rangeeni) during rainy (katki)} crop: Settlement density was more with lesser initial mortality on $C$. calothyrsus $(75.76$ crawlers per sq $\mathrm{cm}$ and 33.58 per cent) while more than 50 per cent initial morality was recorded on $D$. assamica, $C$. surinamensis and $M$. penduliflorus (Table 2). Optimum sex ratio recorded was

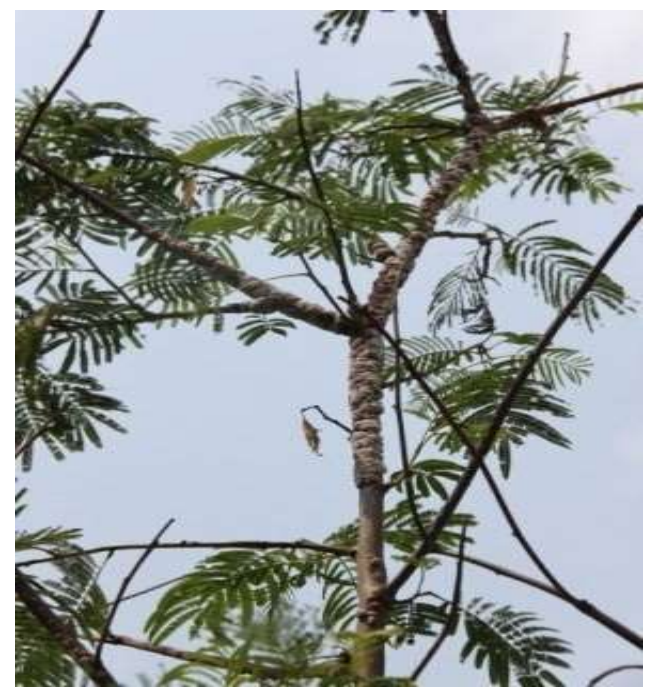

Fig. 4. K. lacca (kusmi) on C. calothyrsus during summer (jethwi) crop

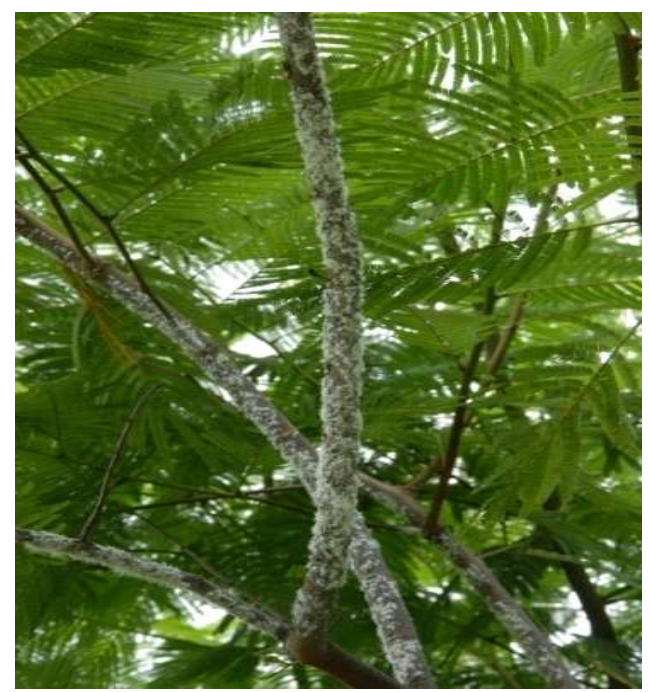

Fig. 5. K. lacca (kusmi) on C. calothyrsus during winter (aghani) crop 
ranging from 25.94 to 35.50 per cent male except on $M$. penduliflorus (1.59\% male) lac insect died before sexual maturation on $M$. penduliflorus. Average fecundity was significantly higher on C. calothyrsus (211.55) and minimum on C. surinamensis (32.95). Cell weight and resin weight recorded were maximum on C. calothyrsus (17.47 and 12.64 $\mathrm{mg}$ ) and minimum on $D$. assamica (12.62 and $9.72 \mathrm{mg}$ ). Yield attributes, viz.,broodlac produced was recorded maximum on C. calothyrsus $(2937.87 \mathrm{~g})$ and minimum on D. assamica $(153.33 \mathrm{~g})$. No broodlac was obtained from $C$. surinamensis. Rejected lac was obtained from three hosts $C$. calothyrsus, $C$. surinamensis and $D$. assamica. Total scrapedlac was significantly more on $C$. calothyrsus $(555.67 \mathrm{~g})$ followed by $C$. surinamensis and $D$. assamica. Broodlac ratio could be calculated only from $C$. calothyrsus (4.46) and $D$. assamica (1.75). The results of the present study also corroborated with the findings of Meena et al (2019) on biological parameters. Kalahal et al (2017) revealed that the initial density of settlement of crawlers varied in different parts of pigeonpea (Cajans Cajan) during Katki season of rangeeni strain. The cell and resin weight ranged from 6-24 $\mathrm{mg}$ and 4$19 \mathrm{mg}$ per cell, respectively. The highest fecundity and cell weight was recorded from ber (450.6 nos. and $10.12 \mathrm{mg}$ ) and lowest on C. cajan (315.4 and $9.4 \mathrm{mg}$ ) during katki crop whereas it was 525.2 and $14.21 \mathrm{mg}$ on ber and 407 and 13.6 mg on pigeonpea respectively, during baisakhi .

Evaluation of $K$. lacca (kusmi) during summer (jethwi) crop: Settlement density of $K$. lacca (kusmi) varied between 62.98 and 77.78 crawlers per sq $\mathrm{cm}$ on $C$. surinamensis, $M$. penduliflorus, $D$. assamica and $C$. calothyrsus while mortality was less on $C$. calothyrsus $(19.99 \%)$ followed by $D$. assamica, M. penduliflorus and C. surinamensis (Table 3 ). Sex ratio ranged between 36.42 and 45.05 per cent male but sex ratio could not be obtained on $M$. penduliflorus as lac insect did not prefer this host for further development. Fecundity was more on C. calothyrsus (214.92) followed by $C$. surinamensis and $D$. assamica. Major difference was not observed in cell weight and resin weight on $C$. calothyrsus, $C$. surinamensis and $D$. assamica. Broodlac and broodlac ratio was more on $C$. surinamensis (996.00 $\mathrm{g}$ and 2.73 ) followed

Table 2. Bio-economical parameters of rainy season (katki) crop of rangeeni lac insect during 2014-2016 (Pooled)

\begin{tabular}{|c|c|c|c|c|c|c|c|c|c|c|}
\hline \multirow[t]{2}{*}{$\begin{array}{l}\text { Bushy host } \\
\text { plants }\end{array}$} & \multicolumn{3}{|c|}{$\begin{array}{l}\text { Pre-harvest productivity } \\
\text { parameters }\end{array}$} & \multicolumn{3}{|c|}{$\begin{array}{l}\text { Post-harvest productivity } \\
\text { parameters }\end{array}$} & \multicolumn{4}{|c|}{ Yield parameters } \\
\hline & $\begin{array}{l}\text { Settlement } \\
\text { density } \\
\left(\text { Nos. } / \mathrm{cm}^{2}\right)\end{array}$ & $\begin{array}{c}\text { Initial } \\
\text { mortality } \\
(\%)\end{array}$ & $\begin{array}{l}\text { Sex ratio } \\
\text { (\% Male) }\end{array}$ & $\begin{array}{c}\text { Fecundity } \\
\text { (nos./ } \\
\text { Female) }\end{array}$ & $\begin{array}{l}\text { Cell weight } \\
\qquad(\mathrm{mg})\end{array}$ & $\begin{array}{l}\text { Resin } \\
\text { weight } \\
\text { (mg) }\end{array}$ & $\begin{array}{l}\text { Broodlac } \\
\text { (g) }\end{array}$ & $\begin{array}{l}\text { Rejected } \\
\text { lac } \\
\text { (g) }\end{array}$ & $\begin{array}{l}\text { Total } \\
\text { scraped lac } \\
\text { (g) }\end{array}$ & $\begin{array}{l}\text { Broodlac ratio } \\
\text { (Output/ input) }\end{array}$ \\
\hline C. calothyrsus & $\begin{array}{l}75.76 \\
(8.73)\end{array}$ & $\begin{array}{l}33.58 \\
(5.81)\end{array}$ & $\begin{array}{l}35.50 \\
(5.99)\end{array}$ & $\begin{array}{l}211.55 \\
(14.55)\end{array}$ & $\begin{array}{l}17.47 \\
(4.24)\end{array}$ & $\begin{array}{l}12.64 \\
(3.62)\end{array}$ & $\begin{array}{c}2937.87 \\
(53.47)\end{array}$ & $\begin{array}{l}1097.00 \\
(32.03)\end{array}$ & $\begin{array}{l}555.67 \\
(23.48)\end{array}$ & $\begin{array}{c}4.46 \\
(2.22)\end{array}$ \\
\hline C. surinamensis & $\begin{array}{l}64.80 \\
(8.08)\end{array}$ & $\begin{array}{l}81.24 \\
(9.04)\end{array}$ & $\begin{array}{l}25.94 \\
(5.14)\end{array}$ & $\begin{array}{l}32.95 \\
(5.28)\end{array}$ & $\begin{array}{l}5.88 \\
(2.37)\end{array}$ & $\begin{array}{c}4.33 \\
(2.07)\end{array}$ & $\begin{array}{c}0.00 \\
(0.71)\end{array}$ & $\begin{array}{l}493.33 \\
(21.17)\end{array}$ & $\begin{array}{l}105.80 \\
(10.30)\end{array}$ & $\begin{array}{c}0.00 \\
(0.71)\end{array}$ \\
\hline D. assamica & $\begin{array}{l}62.47 \\
(7.92)\end{array}$ & $\begin{array}{l}50.88 \\
(7.15)\end{array}$ & $\begin{array}{l}26.49 \\
(5.18)\end{array}$ & $\begin{array}{l}126.31 \\
(11.24)\end{array}$ & $\begin{array}{l}12.62 \\
(3.61)\end{array}$ & $\begin{array}{c}9.72 \\
(3.18)\end{array}$ & $\begin{array}{l}153.33 \\
(12.38)\end{array}$ & $\begin{array}{l}115.33 \\
(10.57)\end{array}$ & $\begin{array}{l}78.67 \\
(8.89)\end{array}$ & $\begin{array}{c}1.75 \\
(1.50)\end{array}$ \\
\hline M. penduliflorus & $\begin{array}{l}51.07 \\
(7.18)\end{array}$ & $\begin{array}{l}66.17 \\
(8.16)\end{array}$ & $\begin{array}{c}1.59 \\
(1.15)\end{array}$ & $\begin{array}{l}0.00 \\
(0.71)\end{array}$ & $\begin{array}{l}0.00 \\
(0.71)\end{array}$ & $\begin{array}{l}0.00 \\
(0.71)\end{array}$ & $\begin{array}{c}0.00 \\
(0.71)\end{array}$ & $\begin{array}{l}0.00 \\
(0.71)\end{array}$ & $\begin{array}{c}0.00 \\
(0.71)\end{array}$ & $\begin{array}{c}0.00 \\
(0.71)\end{array}$ \\
\hline$C D(p=0.05)$ & 0.41 & 0.78 & 0.65 & 1.94 & 0.59 & 0.47 & 7.07 & 8.75 & 1.81 & 0.09 \\
\hline
\end{tabular}

${ }^{*}$ Figures in parentheses are square root $\sqrt{ }(X+0.5)$ transformation values; Means are significant at $p<0.05$

Table 3. Bio-economical parameters of summer season (jethwi) crop of kusmi lac insect during 2014-2016 (Pooled)

\begin{tabular}{|c|c|c|c|c|c|c|c|c|c|c|}
\hline \multirow[t]{2}{*}{$\begin{array}{l}\text { Bushy host } \\
\text { plants }\end{array}$} & \multicolumn{3}{|c|}{$\begin{array}{c}\text { Pre-harvest productivity } \\
\text { parameters }\end{array}$} & \multicolumn{3}{|c|}{$\begin{array}{c}\text { Post-harvest productivity } \\
\text { parameters }\end{array}$} & \multicolumn{4}{|c|}{ Yield parameters } \\
\hline & $\begin{array}{l}\text { Settlement } \\
\text { density } \\
\left(\text { Nos. } / \mathrm{cm}^{2}\right)\end{array}$ & $\begin{array}{c}\text { Initial } \\
\text { mortality } \\
(\%)\end{array}$ & $\begin{array}{l}\text { Sex ratio } \\
\text { (\% Male) }\end{array}$ & $\begin{array}{c}\text { Fecundity } \\
\text { (nos./ } \\
\text { Female) }\end{array}$ & $\begin{array}{l}\text { Cell weight } \\
\qquad(\mathrm{mg})\end{array}$ & $\begin{array}{c}\text { Resin } \\
\text { weight } \\
\text { (mg) }\end{array}$ & $\begin{array}{l}\text { Broodlac } \\
\text { (g) }\end{array}$ & $\begin{array}{c}\text { Rejected } \\
\text { lac } \\
\text { (g) }\end{array}$ & $\begin{array}{c}\text { Total } \\
\text { scraped lac } \\
\text { (g) }\end{array}$ & $\begin{array}{l}\text { Broodlac ratio } \\
\text { (Output/ input) }\end{array}$ \\
\hline C. calothyrsus & $\begin{array}{l}77.78 \\
(8.83)\end{array}$ & $\begin{array}{l}19.99 \\
(4.48)\end{array}$ & $\begin{array}{l}39.51 \\
(6.30)\end{array}$ & $\begin{array}{l}214.92 \\
(14.67)\end{array}$ & $\begin{array}{l}11.40 \\
(3.45)\end{array}$ & $\begin{array}{c}8.99 \\
(3.08)\end{array}$ & $\begin{array}{l}658.00 \\
(25.33)\end{array}$ & $\begin{array}{l}0.00 \\
(0.71)\end{array}$ & $\begin{array}{l}102.00 \\
(9.94)\end{array}$ & $\begin{array}{c}2.03 \\
(1.58)\end{array}$ \\
\hline C. surinamensis & $\begin{array}{l}62.98 \\
(7.97)\end{array}$ & $\begin{array}{l}51.93 \\
(7.23)\end{array}$ & $\begin{array}{l}36.42 \\
(6.05)\end{array}$ & $\begin{array}{l}201.02 \\
(14.17)\end{array}$ & $\begin{array}{r}11.33 \\
(3.44)\end{array}$ & $\begin{array}{c}9.10 \\
(3.10)\end{array}$ & $\begin{array}{l}996.00 \\
(30.72)\end{array}$ & $\begin{array}{l}58.67 \\
(6.27)\end{array}$ & $\begin{array}{l}70.00 \\
(8.17)\end{array}$ & $\begin{array}{c}2.73 \\
(1.78)\end{array}$ \\
\hline D. assamica & $\begin{array}{l}70.89 \\
(8.45)\end{array}$ & $\begin{array}{l}33.64 \\
(5.83)\end{array}$ & $\begin{array}{l}45.05 \\
(6.74)\end{array}$ & $\begin{array}{l}181.70 \\
(13.47)\end{array}$ & $\begin{array}{l}11.83 \\
(3.51)\end{array}$ & $\begin{array}{c}9.68 \\
(3.19)\end{array}$ & $\begin{array}{l}135.27 \\
(11.42)\end{array}$ & $\begin{array}{l}131.53 \\
(10.91)\end{array}$ & $\begin{array}{l}34.00 \\
(5.82)\end{array}$ & $\begin{array}{c}1.24 \\
(1.30)\end{array}$ \\
\hline M. penduliflorus & $\begin{array}{l}66.36 \\
(8.17)\end{array}$ & $\begin{array}{l}45.21 \\
(6.74)\end{array}$ & $\begin{array}{c}0.00 \\
(0.71)\end{array}$ & $\begin{array}{c}0.00 \\
(0.71)\end{array}$ & $\begin{array}{c}0.00 \\
(0.71)\end{array}$ & $\begin{array}{l}0.00 \\
(0.71)\end{array}$ & $\begin{array}{c}0.00 \\
(0.71)\end{array}$ & $\begin{array}{l}0.00 \\
(0.71)\end{array}$ & $\begin{array}{c}0.00 \\
(0.71)\end{array}$ & $\begin{array}{c}0.00 \\
(0.71)\end{array}$ \\
\hline$C D(p=0.05)$ & 0.57 & 0.68 & 0.73 & 1.19 & 0.19 & 0.16 & 6.93 & 4.16 & 2.08 & 0.26 \\
\hline
\end{tabular}

${ }^{*}$ Figures in parentheses are square root $\sqrt{ }(X+0.5)$ transformation values; Means are significant at $p<0.05$ 
Table 4. Bio-economical parameters of winter season (aghani) crop of kusmi lac insect during 2015-2017 (Pooled)

\begin{tabular}{|c|c|c|c|c|c|c|c|c|c|c|}
\hline \multirow[t]{2}{*}{$\begin{array}{l}\text { Bushy host } \\
\text { plants }\end{array}$} & \multicolumn{3}{|c|}{$\begin{array}{l}\text { Pre-harvest productivity } \\
\text { parameters }\end{array}$} & \multicolumn{3}{|c|}{$\begin{array}{l}\text { Post-harvest productivity } \\
\text { parameters }\end{array}$} & \multicolumn{4}{|c|}{ Yield parameters } \\
\hline & $\begin{array}{c}\text { Settlement } \\
\text { density } \\
\left(\text { Nos. } / \mathrm{cm}^{2}\right)\end{array}$ & $\begin{array}{c}\text { Initial } \\
\text { mortality } \\
(\%)\end{array}$ & $\begin{array}{l}\text { Sex ratio } \\
\text { (\% Male) }\end{array}$ & $\begin{array}{c}\text { Fecundity } \\
\text { (nos./ } \\
\text { Female) }\end{array}$ & $\begin{array}{l}\text { Cell weight } \\
\quad(\mathrm{mg})\end{array}$ & $\begin{array}{c}\text { Resin } \\
\text { weight } \\
\text { (mg) }\end{array}$ & $\begin{array}{l}\text { Broodlac } \\
\text { (g) }\end{array}$ & $\begin{array}{c}\text { Rejected } \\
\text { lac } \\
\text { (g) }\end{array}$ & $\begin{array}{l}\text { Total } \\
\text { scraped lac } \\
\text { (g) }\end{array}$ & $\begin{array}{l}\text { Broodlac ratio } \\
\text { (Output/ input) }\end{array}$ \\
\hline C. calothyrsus & $\begin{array}{l}107.91 \\
(10.40)\end{array}$ & $\begin{array}{l}20.29 \\
(4.56)\end{array}$ & $\begin{array}{l}34.04 \\
(5.87)\end{array}$ & $\begin{array}{l}184.52 \\
(13.58)\end{array}$ & $\begin{array}{l}24.58 \\
(5.01)\end{array}$ & $\begin{array}{l}21.73 \\
(4.71)\end{array}$ & $\begin{array}{c}1606.00 \\
(40.00)\end{array}$ & $\begin{array}{c}2049.00 \\
(45.22)\end{array}$ & $\begin{array}{l}849.67 \\
(29.13)\end{array}$ & $\begin{array}{c}3.52 \\
(2.00)\end{array}$ \\
\hline C. surinamensis & $\begin{array}{l}60.71 \\
(7.81)\end{array}$ & $\begin{array}{l}63.78 \\
(8.01)\end{array}$ & $\begin{array}{l}41.20 \\
(6.45)\end{array}$ & $\begin{array}{l}47.78 \\
(6.94)\end{array}$ & $\begin{array}{l}7.03 \\
(2.74)\end{array}$ & $\begin{array}{c}6.19 \\
(2.58)\end{array}$ & $\begin{array}{c}0.00 \\
(0.71)\end{array}$ & $\begin{array}{l}426.67 \\
(20.40)\end{array}$ & $\begin{array}{l}17.33 \\
(4.17)\end{array}$ & $\begin{array}{l}0.00 \\
(0.71)\end{array}$ \\
\hline D. assamica & $\begin{array}{l}69.36 \\
(8.35)\end{array}$ & $\begin{array}{l}31.71 \\
(5.66)\end{array}$ & $\begin{array}{l}42.39 \\
(6.55)\end{array}$ & $\begin{array}{l}39.73 \\
(6.31)\end{array}$ & $\begin{array}{c}7.99 \\
(2.91)\end{array}$ & $\begin{array}{c}6.95 \\
(2.73)\end{array}$ & $\begin{array}{r}16.00 \\
(3.41)\end{array}$ & $\begin{array}{l}255.33 \\
(15.97)\end{array}$ & $\begin{array}{l}37.67 \\
(6.13)\end{array}$ & $\begin{array}{c}0.08 \\
(0.76)\end{array}$ \\
\hline M. penduliflorus & $\begin{array}{l}85.38 \\
(9.24)\end{array}$ & $\begin{array}{l}55.39 \\
(7.47)\end{array}$ & $\begin{array}{l}0.00 \\
(0.71)\end{array}$ & $\begin{array}{l}0.00 \\
(0.71)\end{array}$ & $\begin{array}{l}0.00 \\
(0.71)\end{array}$ & $\begin{array}{c}0.00 \\
(0.71)\end{array}$ & $\begin{array}{l}0.00 \\
(0.71)\end{array}$ & $\begin{array}{l}0.00 \\
(0.71)\end{array}$ & $\begin{array}{l}0.00 \\
(0.71)\end{array}$ & $\begin{array}{c}0.00 \\
(0.71)\end{array}$ \\
\hline$C D(p=0.05)$ & 0.75 & 0.41 & 0.28 & 0.80 & 0.13 & 0.12 & 2.769 & 3.23 & 1.13 & 0.06 \\
\hline
\end{tabular}

${ }^{*}$ Figures in parentheses are square root $\sqrt{ }(X+0.5)$ transformation values; Means are significant at $p<0.05$

by $C$. calothyrsus and $D$. assamica whereas, total scrapedlac obtained was significantly more on C. calothyrsus $(102.00 \mathrm{~g})$ than others and minimum in D. assamica (34.00 g). Mohanta et al (2014), initial settlement density of crawler varied between $104.62-126.74 \mathrm{no} / \mathrm{cm}^{2}$, sex ratio $1: 3$ and resin output per female was 19.00 to $25.60 \mathrm{mg}$ for summer crop of kusmi strain on kusum and ber plants. Slight variation of results may be attributed to different host plants and climatic condition.

Evaluation of K. lacca (kusmi) during winter (aghani) crop: Settlement density of $K$. lacca (kusmi) was more (107.91 crawlers per sq cm) on C. calothyrsus followed by $M$. penduliflorus, $D$. assamica and $C$. calothyrsus. Initial mortality was less on $C$. calothyrsus (20.29 per cent) than the other three hosts (Table 4). Optimum sex ratio (34.04 to 42.39 per cent male) was from three hosts $C$. calothyrsus, $C$. surinamensis and $D$. assamica except $M$. penduliflorus. Average fecundity (184.52), cell and resin weight (24.58 and $21.73 \mathrm{mg}$ ) were recorded significantly more on $C$. calothyrsus than $C$. surinamensis and $D$. assamica. $C$. calothyrsus produced higher broodlac (1606 g per plant) compared to others and the least on $D$. assamica. Total scrapedlac and broodlac ratio obtained were more on $C$. calothyrsus (849.67 $\mathrm{g}$ and 3.52 per plant) than other three hosts. Sharma and Jaiswal (2011) wherein they reported that the mean density of settlement in rangeeni strain of $K$. lacca is lower than that of kusmi strain. The kusmi strain performed better than rangeeni. Results of the present finding of cell and resin weight are significantly more during kusmi winter season crop, respectively.

Field level evaluation of $\boldsymbol{C}$. calothyrsus: Field level evaluation of $C$. calothyrsus was conducted at farmers' fields at two different villages' viz., Lodama and Benyazara of Ranchi district, Jharkhand during 2017-20 for evaluating its potential for commercial lac cultivation. Kusmi broodlac inoculated on C. calothyrsus provided broodlac (470 g per plant), brood per meter lac encrustation (144 g), broodlac ratio (10.5), scrapedlac weight per meter lac encrustation (45 g), per cent scrapedlac (29\%) during aghani, 2018-19 and 2019-20 at Lodma, Ranchi district. Similarly, broodlac (900 g per plant), Brood per meter lac encrustation (111 g), broodlac ratio (5.5), Scrapedlac weight per meter lac encrustation (21 g), per cent Scrapedlac (19\%) was recorded on C. calothyrsus during aghani, 2019-20 at Benyazara, Angara block, Ranchi. This study was also carried out during rainy season crop, 2019 at Lodma which yielded $214 \mathrm{~g}$ of broodlac per plant with broodlac ratio of 5.35.

\section{CONCLUSION}

Lac cultivation on traditional host plants has its own advantages and constraints. Among the bushy host plants studied, C. calothyrsus has shown tremendous potential in lac cultivation based on broodlac ratio of 3-4 and 5.35-10.5 at Institute Research Farm and famers' fields, respectively. It was found suitable to culture kusmi and rangeeni strains of $K$. lacca. It is an indication that this host would perform better as a commercial host.

\section{REFERENCES}

Gupta RK, Bali K and Ganai SA 2020. Natural occurrence of lac insect, Kerria lacca and its conservation in Jammu and Kashmir. Journal of Entomology and Zoology Studies 8(1): 689-695.

Kalahal C, Swami H and Lekha 2017. Productivity-linked parameters of the Rangeeni strain Lac Insect, Kerria lacca (Kerr) on Pigeonpea, Cajanus cajan (Linn.) at Rajasthan. Journal of Entomology and Zoology Studies 5(3): 1745-1751.

Kumar A, Kumawat MM and Meena NK 2007. Lac host plants recorded from southern Rajasthan and their relative performance. Entomon 32: 129-132.

Meena SC, Sharma KK, Rana BS, Swami H, Lekha and Thamilarashi K 2019. Study of productivity linked parameters of lac insect, Kerria lacca (Kerr) on Flemingia macrophylla. Journal of Entomology and Zoology Studies 7(3): 505-509.

Mohanasundaram A, Monobrullah Md, Sharma KK, Meena SC, 
Verma S, Ramani R 2016. Aprostocetus purpureus, a major parasitoid of Indian lac insect, Kerria lacca (Coccoidea: Tachardiidae). Indian Journal of Ecology 43: 517-521.

Mohanasundaram A, Monobrullah Md, Sharma KK, Meena SC and Ramani R 2016. Lac insect and associated fauna: A Practical Manual. ICAR-Indian Institute of Natural Resins and Gums, Ranchi (Jharkhand). pp 01-42.

Mohanta J, Dey DG and Mohanty N 2014. Studies on lac insect (Kerria lacca) for conservation of biodiversity in Similipal Biosphere Reserve, Odisha, India. Journal of Entomology and Zoology Studies 2(1): 1-5.

Monobrullah Md, Mohanasundaram A, Meena SC, Sweta V and Sharma KK 2016. Host and location mediated variation in life cycle and biological attributes of Indian lac insect, Kerria lacca (Kerr.) Indian Journal of Ecology 1: 169-172.

Ramani R and Sharma KK 2010. Record of natural infestation of the Indian lac insect, Kerria lacca (Kerr) (Coccoidea:Tachardiidae) on Acacia tortilis (Forsskal) Hayne and Calliandra surinamensis Benth. Indian Journal of Forestry 33(2): 189-191.

Received 14 October, 2021; Accepted 28 December, 2021
Sharma KK and Jaiswal AK 2011. Biotic factors affecting productivity of lac insect, pp 63-67. In: KK Sharma and Ramani R (eds). Recent Advances in Lac culture, ICAR-Indian Institute of Natural Resins and Gums, Ranchi, India.

Sharma KK and Ramani R 1999. An update on synoptic catalogue of lac insects (Homoptera: Tacchardidae), The Journal of the Bombay Natural History Society 96: 438-443.

Sharma KK, Kumari K and Lakhanpaul S 2007. Host plant mediated variations in resin producing efficiency of Indian lac insect, Kerria lacca (Kerr) (Homoptera: Coccoidea: Tachardiidae). Entomon 32(3): 203-207.

Sharma KK, Mohanasundaram A and Ramani R 2012. Scope of introducing three new and potential plant hosts of $K$. chinensis for sustained and improved lac yield under Ranchi Condition pp.109-110. In: Raipat BS, Shrivastava AK and Sharma KK (eds), Proceedings of IV International conference on Anthropogenic impact on environment and conservation strategies. November 2-4, 2012. National Environmental Association, Ranchi. 